\title{
DISIDENCIAS CONTRAACADÉMICAS DESDE LAS VANGUARDIAS Y EL DOCENTE DE ARTES DEL S. XXI
}

\section{Rocío Silleras Aguilar}

Investigadora independiente

\section{Resumen}

Este artículo señala al autodidacta como un clásico disidente académico de todos los tiempos y áreas, sea oficialmente docto o no y los grandes beneficios que este tradicional agente subversivo aporta al arte, al conocimiento, la formación e incluso a la academia. Expone su importante valor para la educación del siglo XXI donde las tecnologías de la información y la comunicación, el hazlo tú mismo, lo colaborativo y las licencias abiertas y libres han generado un cambio de verticalidad a horizontalidad educativa y cultural. A la vez muestra numerosos antecedentes artísticos de esta democratización, del cambio de ejes que se produciría en todas las esferas de la vida y de estas prácticas contraacadémicas. Y analiza la 'escuela' del siglo XXI, el papel del alumno y el del docente de artes en la actualidad, momento donde adquiere una especial relevancia.

\section{Palabras clave: AUTOAPRENDIZAJE; APRENDIZAJE COLABORATIVO; TUTORIALES; CREATIVE COMMONS; TIC; DOCENCIA S. $\mathrm{XXI}$}

\section{COUNTER-ACADEMIC DISSIDENCES FROM THE AVANT-GARDE AND THE ART TEACHER OF THE XXI CENTURY}

\section{Abstract}

This article points to the self-taught person as a classical academic dissident of all times and areas, either officially learned or not and the great benefits that this traditional subversive agent brings to art, knowledge, education and even academia. It exposes its important value for the education of the $\mathrm{XXI}$ century where the information and communication technologies, the Do It Yourself, the collaborative work and the creative commons licences have generated a change from educational and cultural verticality to horizontality. At the same time it shows numerous artistic antecedents of this democratization, the change of axes that would take place in all the spheres of life and of these counter-academic practices. And analyzes the 'school' of the 21st century, the role of student and that of the teacher of arts today, when it acquires special relevance.

Keywords: SELF-LEARNING; COLLABORATIVE LEARNING; TUTORIALS; CREATIVE COMMONS; ICT; $21^{\text {ST }}$ CENTURY TEACHING

\footnotetext{
Silleras Aguilar, Rocío. 2018. "Disidencias contraacadémicas desde las vanguardias y el docente de artes del s. XXI“. AusArt 6(2): 137151. D0I:10.1387/ausart.20340
}

\section{AUSART}




\section{AUTODIDACTAS, IMPORTANCIA DEL DIVERGENTE CONTRA NORMALIZACIONES}

El autodidacta es clásico disidente de educaciones formales y academicismos muy habitual en artes. Van Gogh, Gauguin, Munch, Bacon o Basquiat muestran las posibilidades del viaje en solitario. Cardillo y sus hiperrealistas esculturas enseñan que para convertirse en escultor solo necesitas profunda atención, fijarte en quien admiras y obstinación hasta la perfección. Manzoni creó la Mierda de artista (1961) y su dedo fue sumo catalizador que convertía objetos y personas en arte tocándoles, autocapacitándose como ácido crítico del establishment artístico. Borges y Saramago son conocidos literatos autoinstruidos, Mozart, Hendrix o Paco de Lucía músicos, Kubrick y Tarantino cineastas, el técnico Ansel Adams, su sistema de zonas y su exquisito uso de la exposición lo son en fotografía, Ibáñez es ilustrador 'no ilustrado' y muchos actores tienen métodos puramente intuitivos.

Los no domesticados frecuentemente amplían áreas y disciplinas como no lo habrían hecho por lo oficial. Picasso fue autodidacta en gran parte de su escultura, esa libertad y su innovador carácter revolucionaron la disciplina ${ }^{1}$, Zappa al no ser dirigido pudo beber de toda música con igual dignidad haciendo fusiones insólitas. Independiente hasta del popular hipismo, solo necesitó su mente para ser referente psicodélico.

Los docentes corremos el peligro de convertirnos en el 'Maestro' que influencia demasiado o negativamente estropeando imaginaciones, estéticas, libertades y espíritus críticos. Deberíamos advertir lo evidenciado por Foucault al hablar del poder de autoridades con capacidad definitoria. Algo que toda persona e institución realiza alguna vez. Un rol hasta oportuno a veces. Lo interesante es que podemos mitigar su negatividad señalándolo, animando a entender bueno/malo de toda actuación y a potenciarse uno mismo dotando de las herramientas, competencias y habilidades necesarias para superarlos, superarnos, superarse y que sean conscientes para elegir libremente qué, cómo, cuándo, dónde, por qué y para qué aprender, adoptar comportamientos, desarrollar praxis, estéticas, crear o usar todo tipo de tecnología. Algo fundamental hoy que hemos de preparar para profesiones y funciones aún no imaginadas.

A veces la verdad oficial entorpece la evolución del arte y del saber. Establecemos cánones, normas y dogmas sobre correcto/incorrecto, artístico o no. Duchamp inscribió a Fountain (1917) en la exposición de la Sociedad de 
Artistas Independientes de Nueva York pero ese transgresivo orinal provocó rechazo a pesar de cumplir normas. Hoy es aclamado como la escultura más determinante del s. XX.

Y desde hace poco se cuestiona su autoría. La verdad oficial evoluciona con nuevos descubrimientos. Investigadores como Camfield quien vio grandes lagunas en Fountain, Gammel descubridora de la autoría de Lötz, Glyn Thompson que confirmó esas teorías y Spalding aceptan su error e intentan corregirlo ${ }^{2}$, pero muchos autores y museos recelan (Libedinsky 2017) por pérdidas de fama, dinero o por negar su verdad. Solo el Tate menciona esto en su web ${ }^{3}$.

Esta problemática no es exclusiva del arte. La Tierra de plana pasó a redondo ombligo universal, después escorado en la vía láctea, posteriormente ínfima parte del universo, luego del multiverso y hoy se teoriza que solo sea información. El Big Bang se hizo Flash y Bounce. Y la materia se dividió en átomos, partículas, quarks y después se lanzaron hipótesis todavía imposibles de falsear con resonantes cuerdas o ínfimas membranas. Ciencia y academia están llenas de verdades refutadas posteriormente, evolucionan con la continua búsqueda, crítica, debate y posterior demostración. Los docentes necesitan ser conscientes del sesgo, cuya peor consecuencia es la lucha de lo nuevo para convertirse en oficial. La búsqueda de la verdad tras la verdad es otra sublevación contracaadémica realizada por doctos y supuestos legos como Ramanujan.

Foucault señaló muchos aspectos sobre el poder y el prejuicio en múltiples instituciones. Aquí destaco la normalización, las consecuencias de creencias absolutistas sobre términos como 'verdad', 'bello' o 'correcto' (Foucault 1966) y la obligatoria inscripción de cualquier enunciado en su contexto (Foucault 1969) ya que sin eso es imposible entender según qué algo es válido/inválido. Estamos acostumbrados a etiquetar y clasificar. Más allá de su practicidad para dar voz, ordenar y facilitar la adquisición de conocimientos no podemos pretender saberlo todo, obviar los juicios de calificaciones, ordenamientos, contextos o perspectivas y abandonar su superación por autocomplacencia. La continua autocrítica es el potente revulsivo del maestro consciente de que siempre será aprendiz.

Alumnos y docentes tenemos que acostumbrarnos a una hiperactualización sin precedentes y a un profundo cambio de era de la que conocemos inicios pero no finales. Ante esto dotar de los conocimientos, herramientas, com- 
petencias y habilidades para ser autónomo, creativo, consciente y crítico se hacen prioridad.

\section{EJEMPLOS DE VANGUARDIA PARA ANORMALIZARSE}

El arte suele ser avanzadilla y hace tiempo que avisa del interés de la no-etiquetación más allá de esa mencionada utilidad. El disidente arte sonoro es ejemplo. 'Arte de los ruidos', 'música experimental', múltiples definiciones de 'arte sonoro' y otros términos lo acompañan desde siempre intentando aclarar un imposible límite arte-música. Cada uno enfatiza cualidades, fines, materias, territorios o comprensiones témporoespaciales ${ }^{4}$ y su definición seguirá mutando con nuevas propuestas. Apoyo esa multidefinición que determina para asimilar a la par que indetermina a favor de libertades creativas, artísticas y autocríticas. Así arte, historia, crítica y palabras evolucionan.

Esta multidisciplinar praxis nacida de las primeras vanguardias y la música experimental lleva a juicio a todo lo oficial y sus poderes, sus calificaciones, lo entendido como arte, obra, artista, público o galería, los límites de disciplinas, medios, lo considerado estético, musical, plástico, lingüístico, escénico, sensorial, la hegemonía ocular, la luz del conocimiento y en suma a toda estructura o agente que ejerce poderes definitorios conduciendo lenguajes, cuerpos, mentes y espíritus. Es una de las prácticas más desarrolladas en los siglos XX y XXI por su unión con lo tecnológico, la universalidad de lo sónico y haber ampliado las materias plásticas, fagocitando a tecnologías, artes, humanidades y ciencias. Esto lo dota de un potencial educativo destacado frente a otras praxis al ser adaptable a las diversas y complejas necesidades del s. XXI.

\section{BENEFICIOS DE CONTRAATACAR LO OFICIAL Y DEL MAESTRO ABIERTO}

Es importante rebasar lo andado. La hiperrepetición de lo normalizado no desarrolla ni conocimiento ni creatividad. Rodin renegó lo aprendido iniciando 
el camino de la escultura moderna, el futurismo insertó el ruido musical y junto al dada extendieron a música, plástica, literatura y escena hasta los confines del universo sónico. El antiarte de Duchamp (y Lötz, todavía no atendida oficialmente) es paradigma de antiacademicismo hoy ineludible en la academia. Mientras el Art Brut acuñado por Dubuffet puso el foco en locos, criminales e infantes quienes fueron maestros libres de ataduras. Atender lo inapropiado, diferente o excluido es otra rebeldía que transforma cánones, modos de vida, prejuicios y aporta conocimiento y nuevas vías.

También es beneficioso abrirse a otras áreas. Proyectar nuevos enfoques y superar fronteras amplía el saber. La historia de la psicología es ejemplo del culto autodidacta humanista y de las posibilidades de reparar en otras esferas para innovar ${ }^{5}$. Mientras Xenakis, oficialmente ingeniero sin formación musical continua, ortodoxa, ni profunda en temas básicos es de los compositores más relevantes del s. XX.

En algunos círculos componer sin dominar lenguaje musical, armonía y piano es sacrilegio. Las escuelas oficiales de música se llaman conservatorios y creo que academias e universidades de todo tipo deberían adoptar términos similares ya que eso hacemos, conservar lo que se produjo antes fuera. Solo algunos profesores extraordinarios batallan contraacadémicamente trayendo lo todavía no aceptado. Ivan Illich es un singular pensador que apostaba por la descolarización y hoy la UAM de Morelos difunde sus escritos y anima a su estudio ${ }^{6}$. Preservar es una acción protesta fundamental contra el olvido dando voz a lo no escuchado haciendo que perdure.

Xenakis encontró un maestro de amplias miras, Messiaen le animó a seguir su camino permitiéndole desarrollar su idea de música y grafías musicales únicas. $Y$ todos recordamos a esos docentes que supieron despertar curiosidades, ampliar perspectivas y avivar intereses, aquellos que ganaron poder respetando, liberando, animando a ser, mostrando la diversión de la aventura del saber y las potentes armas que son conocimiento y estudio. Con Messiaen creció como músico y tuvo una influencia mayúscula en música, arquitectura y plástica como muestra su preludio al Poema Electrónico (Varese) y el Pabellón Philips en el que se proyectó (Le Corbusier y Xenakis, Exposición Universal de Bruselas 1958).

Pervertir, maltratar o asesinar pianos fueron técnicas terroristas contra el hito simbólico y jerárquico de la música culta con las que timbres, interpretaciones, armonías, disarmonías, sonidos, ruidos, silencios, instrumentos y músicas se 
ampliaron, evolucionaron o nacieron hasta lo que son hoy. Las perversiones de instrumentos de Panach Ramos (1903) y su sistema de tercios de tono, las desafinaciones de Ives (Three Quarter Tone Piano Pieces, 1923-1924) y sus clúster con maderos (Sonata para Piano N², 1909-1915), La trampa de la medusa (Satie 1913 o 1914), las interpretaciones de Cowell, el Piano preparado (Cage 1938), la Incidental Music de Brech, la música a distancia de Tudor o Actum, el azaroso absurdo cómico lúdico y cotidiano juego musical de las acciones Fluxus, las propuestas de Canon, Zaj y otros tantos, las músicas rotas, infinitas y de silencio (para nombrar otros medios, instrumentos y músicas), el Paraphrase (Panhuisen y Goedhart 1984), los 3 Fluxus Pianos (Vostel 1993-1994), las instalaciones de pianos intervenidos de Santos o los pianos reconfigurados de Trimpin son ejemplos artísticos contraacadémicos hoy aceptados y ejecutados oficialmente, como el Suicidio de un piano (recreacción por el LCI-UPV en 2012 de una fantasmagoría de Gómez de la Serna publicada en 1935).

\section{ANTECEDENTES ARTÍSTICOS DE HORIZONTALIDAD CREATIVO-EDUCATIVA Y SU CONTINUACIÓN}

El mundo avanza aceleradamente y con él arte, ciencia, educación y academia. Todo docente necesita comprender que sobre todo hoy somos prescindibles. La escuela del s. XXI se llama internet, no oficial pero con más alumnos que ninguna. Llena de canales educativos, tutoriales en línea, prácticas 'hazlo tú mismo' (Do It Yourself o DIY), cursos en línea masivos y abiertos (Massive Online Open Courses o mooc), plataformas de formación en línea (e-educación), sistemas operativos, softwares y hardwares libres (como Linux, Arduino o Puredata) y con un patrimonio cultural común extendiéndose, ampliándose y compartiéndose gracias a licencias de autor libres (creative commons o c.c.). Todo esto fomenta un nuevo modo de aprendizaje colaborativo donde el maestro todopoderoso no cabe. La jerarquía educativa está cambiando por las tecnologías de la información y la comunicación (TIC). Debemos repensar nuestra función como el arte del s. XX hizo.

Los anteriores ejemplos artísticos muestran antecedentes del cambio, un paso de vertical a horizontal y de individual a colectivo y colaborativo. Enseñan esa democratización y evolución del DIY, las licencias libres y la colaboración en 
las artes durante todo el siglo, siendo referentes artísticos de lo por venir en todas las esferas del conocimiento y la vida.

En la escucha de los paisajes sonoros del mundo con el máximo exponente del 4'33" (Cage 1952) todos los ruidos y sones son música y un oído atento basta para componer la propia interpretación de la mayor sinfonía creative commons que jamás existirá. En música experimental el uso de lo cotidiano, del ruido musical, el azar, la indeterminación, lo abierto y esa libertad interpretativa apta para músicos y nomúsicos democratizó y popularizó la música culta. Los nuevos modos de tocar, la preparación de instrumentos y el Circuit Bending que le continúa (práctica experimental con la que jugando y transformando aprendes electrónica y desarrollas instrumentos nuevos) son propuestas contraacadémicas DIY y una inteligente forma de 'Aprender a Aprender' e innovar basadas en el juego experimental (en el sentido de play o jouer, jugar y tocar un instrumento) con metodologías provenientes de las artes plásticas y la música experimental de gran utilidad educativa hoy.

Un conocido 'hazlo tú mismo' artístico son las instrucciones Para hacer un poema dadaísta (Tzara 1920) con las que artistas y anartistas pueden encumbrarse como poetas del antiarte. ${ }^{7}$ Los ready made evidenciaron que apropiación y sacar de contexto bastan para elevar urinarios a la categoría de arte sin necesidad técnica. La huella de Manzoni creó arte con un gesto replicable por cualquiera. $Y$ con Fluxus, Zaj y otros fluxartistas hoy vida=arte y arte=vida. Estos ejemplos muestran que el pensamiento crítico y el divergente son grandes aliados para crear e innovar.

La Poesía Blackout que muchos creen un DIY contemporáneo tiene raíces en las vanguardias e incluso antes ${ }^{8}$. Técnicas agresivas como la ruptura, el corta-pega, tachado, borrado, quemado, frotado, agujereado, explotado... tan típicas de vanguardia desafían el control de academias, lenguajes, imágenes, objetos, artes y músicas construyendo por destrucción. Sublevaciones hoy populares más allá de la escuela por la seducción de lo inapropiado, el juego y el descubrimiento de lo experiencial.

Otras obras actuales con potencial educativo de interés para el s. XXI son aquellas en las que las TIC amplían los procesos de creación, mecenazgo, producción y difusión, en las que un 'público' consciente o inconsciente se hace partícipe, colaborador y hasta creador. En "Universos" (3.257 internautas, José Corbacho y Juan Cruz, 2009) y "Miedo" (6.034 internautas y Jaume Balagueró, $2010^{10}$ ) miles de personas codirigieron con conocidos profesiona- 
les. El Cosmonauta (Nicolás Alcalá 2008-2013 ${ }^{11}$ ) es un proyecto transmedia que enseña las bondades y miserias del micromecenazgo, lo colaborativo y lo c.c.; Y el documental Hard as Indie (Arturo Antolín, 2017 ${ }^{12}$ ) analiza ese experimento que trató de cambiar el status quo de nuestro cine. Con ellos podemos mostrar el potencial de las formas de hacer actuales.

\section{ARMARSE ARTÍSTICAMENTE FRENTE A MANIPULACIONES CONTEMPORÁNEAS}

Estas y otras negaciones DIY llevadas al extremo permiten destrozar mecanismos, estructuras y sistemas. Las poesías ruidistas más radicales son ejemplo. Cuando dejas solo ruidos se rompe hasta la jerarquía melódica quedando lo no dirigente ni informativo, pero solo en partitura permanecerán totalmente abiertas y libres de interpretación ${ }^{13}$. $Y$ es que las estrategias contrasistema también pueden redefinir realidades revirtiendo poderes, transformando al manipulado en manipulador.

En "La revolución electrónica" (1970) Burroughs señaló la importancia del cut-up (corte), la edición en general y su potencia terrorista. Manipulaciones negativas son los cotidianos fakes (fake news, noticias falsas) con intereses políticos, económicos, sociales... que proliferan en las redes. Frente a los que deberíamos enseñar a discernir y criticar contraatacando con una actitud crítica madura, el interés por la búsqueda de buenas fuentes y el contrastar información para verificar.

Eres lo que lees (Guillermo Vargas Jiménez, "Habacuc", 2007) es una obra tan mundialmente conocida como desconocida al basarse en la posverdad (mentira emotiva). De él solo recordamos el falso título de maltratador de perros que en realidad fue difundido por Habacuc. Con las TIC recreó una pieza con la reacción inconsciente de las redes aprovechando la bondad del usuario para hacer emerger la hipocresía de individuos, sociedades y establishments artísticos, mediales, tecnológicos y sociales. Metió el dedo en una llaga relevante hoy, aprovechando la capacidad manipuladora de estas deformaciones deliberadas de la realidad que modelan la opinión pública mediante una emoción que supera a la verdad. Una artística falsedad con la que podemos analizar las consecuencias de estas mixtificaciones abarcando temas como ciberacoso, ciberactivismo, censura 2.0 y el inteligente uso de las redes. 
Mientras manipulaciones positivas aparecen en los demiurgos libertarios no-dirigentes, capaces de entresacar un misterio del mundo recreando otro a través del recorte y la negación del anterior, permitiendo que uno sea el único intérprete. Un ejemplo contemporáneo de desvelamiento por borrado es la aplicación The Deletionist (Amaranth Borsuk ${ }^{14}$, Jesper Juul ${ }^{15}$ y Nick Montfort ${ }^{16}$, 2013) ${ }^{17}$ que entresaca poemas escondidos en toda web.

\section{DESARROLLAR LA LIBERTAD- RESPONSABILIDAD MEDIANTE EL PLACER DE APRENDER}

Vivir ya es autoinstruirse. Formados oficialmente o no, conscientes e inconscientes todos somos autodidactas. En el deglutir lo oficial, analizar y ejercitar lo social en redes, aceptar fakes, afrontar manipulaciones o seleccionar música estás eligiendo formación. El autoaprendizaje se señala como el futuro, pero siempre fue presente porque aprender es vital, individual, intransferible y avanza con tus decisiones.

Solo los que buscaron más allá pudieron superarse para crear algo nuevo y ahora creatividad e innovación están en el centro de la educación. Zappa duro poco en la universidad y formó a sus hijos con independencia. Prefería una autoformación que ejercitase el pensamiento crítico propio. Bibliotecas y calles fueron sus aulas. Cuentan de Cage que en Pomona "Experimenta preparar los exámenes cogiendo a ciegas libros distintos de los recomendados. Saca con ese método la mejor y la peor nota... ¿Luego estudié sólo con personas cuyas ideas me interesaban...)" (Citado en Matamoros \& Aracil 2013). Su inortodoxia no aportó matrículas oficiales pero les dio perspectivas que ampliaron su comprensión de lo musical. Ese ir más allá por gusto hace que sean recordados.

El mayor mentor de Einstein fue el surtido de ánimos y libros científicos de su tío. Einstein señaló al placer como el mejor método, "Esa es la mejor manera de aprender, cuando estás haciendo algo con tal disfrute que no te das cuenta de que el tiempo pasa" (Mc Cullough 2004). Lo oficial es dispensable, curiosidad y pasión no. 


\section{BENEFICIOS DE LA EDUCACIÓN ARTÍSTICA PARA LA NUEVA EDUCACIÓN}

Da Vinci es paradigma de arte, ciencia, hiperproductividad multidisciplinar renacentista y de los beneficios de la curiosidad transdisciplinar y del autoaprendizaje que orina fuera de tiestos. Comenzó por su cuenta dibujo, pintura y escultura y aprovecho las enseñanzas multidisciplinares de Verroquio y Pollaiuolo a quienes pronto superó. Su gran escuela fue la vida que escudriñó incansablemente. Realizó dibujos, pinturas, esculturas, arquitecturas, escenografías, estudios urbanísticos, musicales, botánicos, anatómicos, armamentísticos, metalúrgicos y en mecánicas e ingenierías de todo tipo. Hasta las manchas de las paredes le inspiraron y señala su observación como método para autodesarrollar la creatividad. Su mayor genialidad fue aunar artes, ciencias y vida en su autoinstrucción. Él es el perfecto ejemplo de la fuerza de su suma. Una reunión que reaparece con la Educación STEAM (Science, Technology, Engineering, Arts and Mathematics) que evidencia la potencia educativa de las artes para facilitar ciencias exactas y a lo que sumar las sociales.

Leonardo también sirve para introducir a científicos autodidactas como Nikola Tesla o Srinivasa Ramanujan (matemáticos), Richard Buckminster Fuller (diseñador, arquitecto e inventor atitulado) o Steve Jobs (Appel), a artistas-científicos como Goethe o Lewis Carroll y a científicos-artistas como Samuel Morse (pintor), Richard Feynman (Nobel de Física, dibujos de Ofey), el conocido Queen Brian May (astrofísico) o el mismísimo Albert Einstein (músico). Einstein creía que los buenos científicos eran artistas, seguramente por las alas que da la libre imaginación. Parece que la reunión música-universo y su espiritualidad marcaron sus revolucionarias teorías. Mientras Xenakis creó la estocástica con la influencia de la ciencia (matemática, gráfica, procesos transformativos...). Las artes fomentan creatividades y facilitan conocimientos complejos a través de prácticas placenteras que usan vías más accesibles y no trilladas por las ciencias.

\section{FUNCIONES BÁSICAS DEL ALUMNO Y DEL DOCENTE DE ARTES DEL S. XXI}

La cambiante actualidad, la imparable globalización e hiperrevolución tecnológica llena de TICs, IdCs (Internet de las cosas), 'inteligencias' artificiales, 
industrias 4.0 y la popularización de impresoras 3D hacen que el ágora, la escuela y hasta los espacios de producción se establezcan en casa. Resulta obligatorio un 'Aprender a aprender' actualizado y apto para el s. XXI. Hoy conceptos como análisis crítico, imaginación, creatividad y pensamiento disruptivo son clave y mañana cuando lo único que se mueva de un lado a otro sean ideas serán lo único a ofrecer. Los profesores de artes como avivadores de críticas, imaginaciones, autogestiones y rupturas nos encontramos en un lugar relevante.

Internet aporta infinidad de contenidos pero no enseña cómo moverse en sus aguas, ni desarrolla un pensamiento crítico y creativo propio, maduro, consciente y autónomo. Esa me parece nuestra función fundamental actualmente, potenciar al insurrecto autodidacta y al creativo que llevamos dentro para que evolucione con independencia on y offline. Recordando su extensión por la web ${ }^{18}$ y sin olvidar lo 0.0 (prehistoria e historia previa a internet) porque antes ya existían el placer, la curiosidad, el autoaprendizaje y sus originales metodologías.

La del alumno es adquirir esa ineludible responsabilidad y con ella los conocimientos, competencias y habilidades que le permitan su independencia y libertad. Hemos de fomentar la asunción de este compromiso y aportar lo necesario para desarrollar mejores autodidactas con mayor consciencia, control y potencia para desarrollar su aprendizaje el resto de su vida. La actual hiperactualización apremia. Solo ellos podrán autocapacitarse para lo por venir.

\section{APOYAR A DIVERSOS PERFILES PARA MAYOR VARIEDAD DE AUTODIDACTAS}

Ahora en esa reunión de arte y ciencia y su valor educativo hasta se habla de los "T-shaped"19 y los "pi-shaped science artist"20 (Harris 2016). Estoy de acuerdo en la conveniencia de intentar potenciar sobre todo a estos últimos, pero no en centrarnos solo en ellos. Desconozco si la afirmación de Einstein de que los mejores científicos son obligatoriamente artistas es totalmente cierta, pero sí sé que todos no somos genios, ni que todos los grandes artistas han sido expertos científicos. Aún así su imaginación vislumbró cosas que otros no pudieron soñar y habitualmente sus visiones plantan la semilla de propuestas científicas y tecnológicas de futuro. El aerodeslizador de Marty 
McFly en Regreso al futuro (película de Robert Zemekis, 1985) es desde 2015 un sueño hecho realidad desarrollado por Lexus (proyecto Slide).

Seguramente los genios son del tipo pi-shaped, quienes como Da Vinci eran expertos en artes y ciencias causando alto impacto en ambas. Pero los artistas, científicos y otros profesionales $T$-shaped, doctos en un área y capaces de enriquecerse de sus aproximaciones a otras formulan ideas que los anteriores por sapiencia desdeñan. Zappa concebía su música como una escultura o arquitectura, muchas acciones y fluxperformances son músicas o amúsicas, las esculturas de los Baschet instrumentos musicales y los Headscapes de Leitner (1987-2003) paisajes sonoros de estructuras arquitectónicas para recorrer mentalmente. Aunque esas ideas no tuvieran relevancia en esas otras esferas sí hicieron avanzar la propia.

La música se compone de sonido, ruido y silencio. Sin ellos no es. Y precisamente fue el inculto ruido lleno de disarmonías el que revolucionó la música del siglo XX. Algo similar ocurre con la ciencia y el conocimiento que necesitan de prueba, acierto/error y se pueden hacer evolucionar con el disparate. El azar, la serendipia y lo aparentemente equivocado también han hecho avanzar al conocimiento. La penicilina es ejemplo afamado mientras la controversia de la fallida teoría del todo de Garrett Lisi o el uso del Glitch en arte son actuales.

Hemos de alentar y apoyar que alcancen su máximo potencial, pero esa máxima no siempre es la genialidad. Deberíamos nutrir a cada autodidacta según su naturaleza, capacitarles para erigirse por sí mismos en expertos de lo que deseen. Así tendremos más perspectivas que aviven reflexiones críticas y amplíen horizontes de comprensión. Del punto sale la línea, de ella el plano y con ellos el volumen. Como docentes hemos de beneficiar y beneficiarnos de todos. El conocimiento se desarrolla en comunidad, más hoy con la hipermente conectada y aquí la diferencia suma.

\section{Referencias}

Burroughs, William Seward. (1970) 2005. The electronic revolution. New York: UBU. https:// www.swissinstitute.net/2001-2006/Images/electronic_revolution.pdf

Foucault, Michel. (1966) 2007. Las palabras y las cosas: Una arqueología de las ciencias humanas. Traducción de Elsa Cecilia Frost. Madrid: Siglo XXI

Foucault, Michel. (1969) 2009. La arqueología del saber. Traducción de Aurelio Garzón del Camino. Madrid: Siglo XXI 
Harris, David. 2016. "The need for more pi-shaped science artists". Clot Magazine, 10 ago. http://www.clotmag.com/pi-shaped

Howarth, Sophie. (2000) 2015. "Marcel Duchamp: Fountain 1917, replica 1964". Tate.org. Revised by Jennifer Mundy. https://www.tate.org.uk/art/artworks/duchamp-fountain-t07573

Lawson, Dorie McCullough. (2004) 2008. Posterity: Letters of great Americans to their children New York: Anchor

Libedinsky, Juana. 2017. "Duchamp, la fuente y el inodoro de oro”. La Nación, 29 ene. https:// www.lanacion.com.ar/1979357-duchamp-la-fuente-y-el-inodoro-de-oro

López de Benito, Ramón. 2016. "Picasso: Esculturas en papel para un nuevo pensamiento estético." Boletín de Arte [UMA Dpto. $\mathrm{H}^{\mathrm{a}}$ Arte] 37: 129-38

Matamoros Ocaña, Elena \& Alfredo Aracil Ávila. 2013. "Cage 101." Por la Danza 98: 84-9. http:// www.alfredoaracil.info/docs/writings/Cage101PorlaDanza.pdf

Mora Suárez-Varela, Vicente Luis. 2015. "Arte y tachado". Diario de lecturas, blog personal, 28 abril. http://vicenteluismora.blogspot.com/2015/04/arte-y-tachado.html

\section{Notas}

${ }^{1}$ Con sus estudios de guitarras supuso un cambio en los procesos escultóricos que liberaron de las maneras tradicionales como el modelado y la talla, así como de conceptos clásicos como el volumen, lo que supuso toda una revolución en la disciplina escultórica. Resulta que fue Braque quien inició en 1911 este tipo de estudios escultóricos para aplicarlos a su pintura pero según López de Benito $(2016,130)$ "Braque no pareció darles mucha importancia y finalmente terminaron por desaparecer... solo se conserva como referencia la fotografía (fig.1: Construcción de Braque realizada en el Hotel Roma, primavera de 1914)". Picasso sí le dio valor siendo finalmente el que las introduciría oficialmente en la escultura ya que dio mayor importancia a esos estudios en papel e incluso realizó una versión de Guitarra en metal (ibíd. 130-1).

${ }^{2}$ Error y corrección a la que me sumo ya que también aprendí que Duchamp era el autor oficial.

${ }^{3}$ https://www.tate.org.uk/art/artworks/duchamp-fountain-t07573

${ }^{4}$ Comenzó con conceptos como 'arte de los ruidos' (Russolo 1913) y aunque ya era una forma de música experimental proveniente de un artista plástico y músico, y su fin era musical esa terminología no se popularizó hasta más tarde. En música culta oficial Messiaen y sus usos de las ondas Martenot (desde 1937 aprox.) son otro claro referente de los inicios de la música experimental y electrónica, aunque desconozco si él lo entendía como tal o simplemente como música. El término se popularizó con la música electroacústica y con Cage (años 50). También le siguieron otros términos desde la música (electroacústica, acusmática, concreta, estocástica...) y desde la plástica (anti-música fluxus, a-música de Nam June Paik, música abierta de Hidalgo y no-música de López). Mientras el término 'arte sonoro' (Sound Art, Audio Art, Art Sonore, Klangkunst) proviene de la plástica (primeros referentes 1983 Exposición Sound/Art, The Sculpture Center N.Y.) en parte para incluir obras que no cabían en la categoría de música como las de la poesía experimental, performance y del arte visual. Proliferó mucho por la conveniente libertad que aporta apelar a lo sónico para ir más allá de lo musical. Pero esa adjetivación que destaca el uso del sonido como elemento principal de la pieza, muchas veces es entendida etimológica o literalmente dejando fuera infinidad de obras que se relacionan directamente con lo sónico 
y lo vibratorio, o aquellas que aun siendo profundamente sonoras no tienen al sonido como elemento central destacado o no es lo sónico su fin último. Además los intonarumori son instrumentos musicales ruidistas que forman parte de él y ha llegado a fagocitar todo tipo de praxis artísticas, musicales, cuasimusicales, literarias, escénicas... bajo su denominación. Y la definición de arte sonoro ha variado mucho con los años hasta llegar a la indefinición. Las palabras evolucionan con su uso, desuso o abuso y al arte sonoro le han seguido el 'arte de la escucha', la 'transdisciplina', el 'intermedia', 'media art' y muchos otros. En 'intermedia' Higgins ya lo señaló como mera herramienta para entender experiencias artísticas que se encuentran entre medios tradicionales y que al atender a esos medios hay que ir más allá de técnicas y materiales. Y si pensamos en posibles antecedentes del arte sonoro aparecen infinidad de ejemplos históricos y prehistóricos que dificultan aún más la tarea.

${ }^{5}$ Freud fue un neurólogo fundador del psicoanálisis, el filósofo William James creó la psicología funcional, Wundt un fisiólogo pionero en psicología social que creó el primer laboratorio de psicología experimental revolucionándola hacia lo científico, Piaget siendo doctor en ciencias naturales (biología) inició la psicología evolutiva y Vygotsky (oficialmente estudió algo de derecho, filosofía y letras) fundó la psicología histórico-cultural, es antecedente en neuropsicología e hizo grandes aportes a la psicología pedagógica.

${ }^{6}$ Ivanillich.org.mx, web que compila la obra de Iván Illich, mantenida por Braulio Hornedo (Universidad Virtual Alfonsina Cuernavaca). http://www.ivanillich.org.mx/

${ }^{7}$ Estas directrices son una forma de disidencia contraacadémica y contraartística al permitir que cualquiera se establezca como poeta dada recortando palabras de un artículo periodístico, mezclando esos recortes y sacándolos al azar para que sea este el verdadero autor de una pieza enigmática. Es el establecimiento de un nuevo mundo por el desvelamiento de lo oculto en el que cada persona obtendrá una interpretación propia. Democratiza el papel del artista, otorga valor significante al escucha y torpedea la idea de arte elevado mediante su antítesis usando al misterio y lo incomprensible irónicamente.

${ }^{8}$ Aparece en las acciones neodadá de Fluxus (60's hasta hoy), en las chamánicas y curativas cruces de Beuys, en las del Tachismo (50's) que construyen a través del juego construcción-destrucción y ruptura-unión, en las de Tapies y su Informalismo (50's) o en el antipoema de Man Ray Poema (1924). Hasta Heidegger realizó una cruz en forma de $x$ sobre la palabra 'Ser' en la carta "Hacia la pregunta del ser" (1956) una respuesta a "Sobre la línea" de Ernst Jünger, textos en donde analizaban el nihilismo en base a La voluntad de poder (1901) de Nietzsche (Mora [2013] 2018).

9 "Universos", vídeo ganador de la $1^{\text {a }}$ ed. de La Wikipeli de Mahou (2009), dirigido por José Corbacho, Juan Cruz y 3.257 internautas. 30:09. https://www.youtube.com/watch?v=rdS_ pgDLGXA

10 "Miedo", vídeo ganador de la $2^{\mathrm{a}}$ ed. de La Wikipeli de Mahou (2010), dirigido por Jaume Balagueró y 6.034 internautas 25:26. https://www.youtube.com/watch?v=rdS_pgDLGXA

11 "El Cosmonauta" (2013), dirigida por Nicolás Alcalá. Riot Cinema Collective. Video de Youtube, 1:42:16. https://www.youtube.com/watch?v=yqFXe2EnHnE

12 "Hard as Indie" (2017), video dirigido por Arturo M. Antolín 1:32:56. https://hardasindie.com/ pelicula

${ }^{13}$ Cuando te quedas únicamente con las vocales de un texto rompes ciertas estructuras pero no la jerarquía melódica establecida por ellas. A veces puedes intuir el significado que tenía la palabra por entonación. El silbo gomero se basa en esto. Pero cuando te quedas únicamente con consonantes (ruido) o con ruidos y pocas vocales inconexas se rompe toda jerarquía y estructura, solo queda lo superfluo, lo no dirigente y lo no-informativo. 
Aquí rompes el sistema de control lingüístico pero la interpretación que hagas y tu lenguaje no verbal volverán a dotarles de sentido, sin embargo, en su partitura sí permanecerán no-dirigentes, abiertas y libres para cualquier otra interpretación.

${ }^{14}$ Amaranth Borsuk, web personal. http://www.amaranthborsuk.com

${ }^{15}$ Jesper Juul, web personal. http://www.jesperjuul.net

${ }^{16}$ Nick Montfort, web personal. http://nickm.com/

${ }^{17}$ En sus webs se pueden encontrar numerosas propuestas de poesía disidente contraacadémica por medios digitales y analógicos. https://thedeletionist.com/

${ }^{18}$ Estática (1.0), social y dinámica (2.0), semántica (3.0), ubicua (4.0) y hasta sensorial (5.0).

${ }^{19}$ Artistas-científicos de perfil en forma de T, saben poco de muchas cosas y mucho de arte o de ciencia pero no de las otras disciplinas que no son su base, pudiendo solo causar impacto en la que son doctos.

${ }^{20}$ Artistas-científicos de perfil en forma de pi, saben mucho de arte y ciencia, tienen competencias y habilidades en ambas y pueden causar un profundo impacto en ambas.

(Artículo recibido: 06-11-18; aceptado: 07-01-19) 